\title{
MANAGEMENT OF LIMB INJURIES
}

\author{
Keith M Willett, Hugh Dorrell, Peter Kelly
}

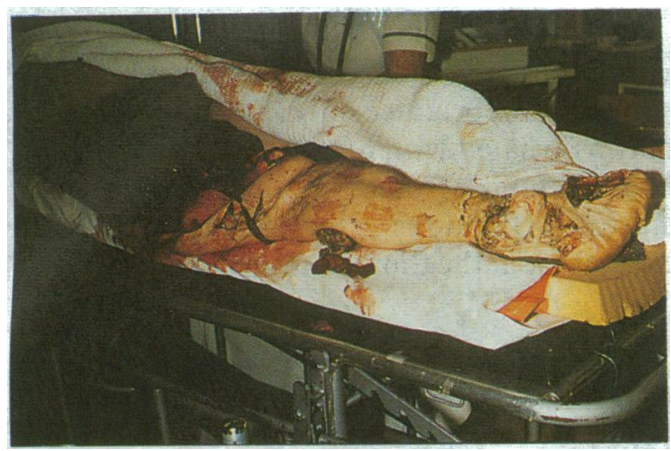

\section{Prehospital care}
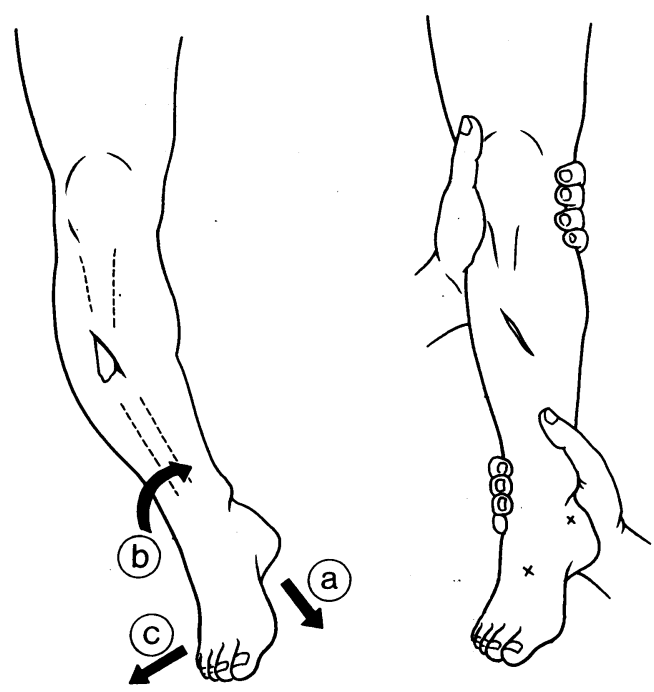

(1) Reduction of a major deformity; (a) perform gentle longitudinal traction) (b) restore the correct rotation; (c) restore the alignment.

(2) Control of the fracture; check pulses $(x)$; maintain traction; apply a splint.

\section{Hospital care}

\section{Estimated blood loss caused by fractures}

\begin{tabular}{lc}
\hline Site of fracture & $\begin{array}{c}\text { Blood loss } \\
\text { (litres) }\end{array}$ \\
\hline Humerus & $0.5-1.5$ \\
Tibia & $0.5-1.5$ \\
Femur & $1 \cdot 0-2.5$ \\
Pelvis & $1 \cdot 0-4.0$ \\
\hline
\end{tabular}

For an open fracture the loss is two or three times greater.
Up to $70 \%$ of multiply injured patients have injured limbs and fractures or dislocations of the appendicular skeleton. Severe limb injuries must not distract the resuscitation team from the priorities of establishing an airway, optimising ventilation, and restoring circulatory volume as limb injuries are rarely immediately life threatening, except those that cause exsanguination.

Careful thorough examination, however, is required after resuscitation to identify injuries, particularly those threatening the survival of limbs or fractures whose acute management will influence overall mortality or morbidity. Apparently minor injuries must likewise not be neglected as these may result in long term disability or disfigurement.

Doctors attending the scene of an accident should confine themselves to management of the airway and ventilation and immobilisation of the cervical spine and injured limbs. When moving a patient with a fractured limb the pain is reduced by supporting the limb on either side of the fracture and applying gentle traction along the axis of the limb. All unnecessary handling of the injured part without splinting should be avoided. The exceptions to this rule are when either severe deformity or ischaemia of the limb distal to the fracture threatens survival of the soft tissues; reduction is then indicated. This is achieved by gentle traction and restoration of the normal anatomical alignment. Perfusion of the distal limb must be checked after any manipulation. Prehospital care must avoid further soft tissue injury.

Splints are mandatory before the victim is evacuated; and anything rigid can be utilised - for example, pieces of wreckage, wooden sticks, etc. Strapping to the opposite leg is useful in solitary lower limb injuries, and "bulk" splints can be produced by bandaging blankets or pillows around the limb. Wounds should be covered with a clean dressing, preferably one that is sterile. External bleeding can be controlled by a compressive pad. Rapid transfer to hospital is then required.

\section{Haemorrhage}

Blood loss from limb wounds and occult bleeding from fractures contribute to the hypovolaemic shock in patients with multiple injuries. The accumulative haemorrhage from multiple fractures may result in exsanguination; patients with fractures of the femora and pelvis are at greatest risk. Patients with hypovolaemic shock should be resuscitated immediately with available crystalloid or colloid solutions while the dynamic response of the blood pressure and pulse is monitored. The total blood loss may be estimated (table), and blood for transfusion should be cross matched urgently. Blood loss from open fractures may be two or three times greater than that from closed fractures. Fractures, however, should not be assumed to be responsible for hypovolaemia, and occult bleeding into the visceral cavities should be excluded. Blood loss from wounds can be reduced by a compressive bandage or hand pressure over a sterile pad. A tourniquet is indicated only for unmanageable life threatening haemorrhage or after traumatic amputation. 


\section{Life threatening injuries}

- Traumatic amputation

- Major vascular injury

- Pelvic fracture disruption

- Haemorrhage from open fractures

- Multiple long bone fractures

- Severe crush injury

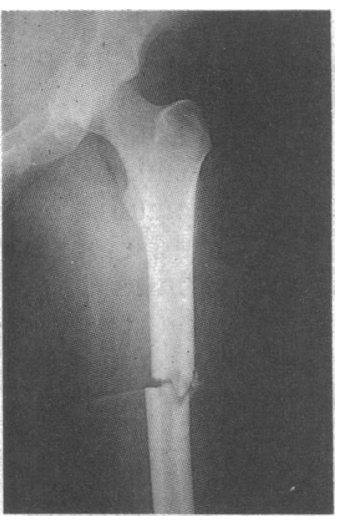

Acetabular fracture associated with a femoral shaft fracture.

\section{Limb threatening injuries}

- Vascular injury

- Major joint dislocation

- Crush injury

- Open fracture

- Compartment syndrome

- Nerve injury

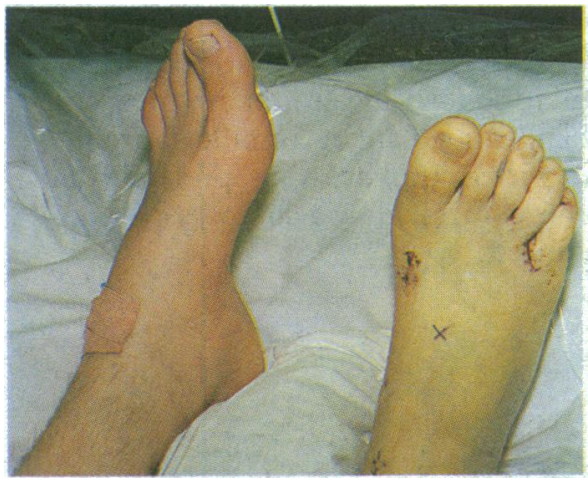

Ischaemic right foot.

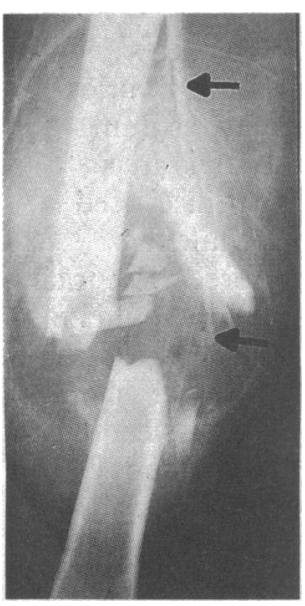

Femoral shaft

fracture.

Arteriogram

showing occlusion

of the superficial

femoral artery at

the level of the

fracture.

\section{Assessment}

Evaluation of limb injuries is not started until the life threatening conditions have been treated. Careful examination is complemented by suspicions raised by knowledge of the mechanism of injury, information on which may be available only from attendants present at the scene of the accident. These witnesses should not be discharged until at least the mechanism, environment, time, and immediate care of the injury have been established. For victims of road traffic accidents it is important to determine whether they were a vehicle occupant, whether they were restrained by a seat belt, the direction of the impact, and the degree of damage to the vehicle. Ejection from a motor vehicle carries a high risk of serious injury.

Certain injury patterns are common. For instance, a direct blow to the knee in a seated occupant of a car may not produce only knee injury and femoral fracture but is commonly associated with hip dislocation or fractures of the acetabulum. A victim falling from a height and landing on his or her feet may sustain compression fractures of the calcaneum, ankle, tibial plateau, and one or more vertebrae at the thoracolumbar junction or in the lower cervical spine.

The patient must be completely undressed. The assessment should begin by comparing the injured limb with the uninjured limb. Observe the attitude of the limb: shortening and rotational abnormalities indicate proximal fractures or dislocations. Angular or rotational deformity may be visible or palpable. Clinical signs are often subtle, particularly in the unconscious patient, and careful inspection of the whole circumference and length of each limb for local swelling and bruising is necessary. Gently palpate along the axes of the bones and all of the surface bony prominences for tenderness, fracture crepitus (grating), and abnormal interfragmentary mobility. Carefully examine the adjacent joints so that coexisting injuries are not overlooked. A cooperative patient may indicate the active ranges of joint movement. Passive ranges of motion should be assessed cautiously in a limb that is suspected of being fractured; these should not be tested if an obvious fracture exists.

\section{Vascular state}

Of prime importance to limb survival is the competence of the vasculature distal to any injury. Local contusion, penetrating injuries, fractures, and, particularly, major joint dislocations may occlude or disrupt blood vessels. In the haemodynamically stable patient examination of the distal pulses is crucial in assessing the peripheral circulation. A diminished or absent pulse strongly suggests a vascular injury and must be explained and managed promptly. Skin colour will also indicate tissue perfusion, and pallor or a blue-grey colour should arouse suspicion. Similarly, a low skin temperature indicates inadequate perfusion. A sensitive indicator is the capillary return - the normal prompt pink flush of the nail bed seen after transient compression. This response will be slowed or blue if the circulation is inadequate.

Peripheral nerves are very sensitive to ischaemia, and sensation is lost early. Total insensibility in a hand or foot suggests ischaemia as, except in patients with injuries to the brachial plexus or spinal cord, it is unlikely that all nerve trunks will have been damaged primarily in one limb. An inadequate distal circulation is never due to spasm in a traumatised limb. If distal ischaemia is identified more proximal pulses should be checked and any major deformity at the fracture site corrected, the splint device checked for local compression, and an urgent surgical opinion sought. Dislocations of major joints require urgent reduction. Doppler ultrasonography may be useful in evaluating limb perfusion, but if a vascular injury is suspected arteriography provides the best definitive evaluation. 


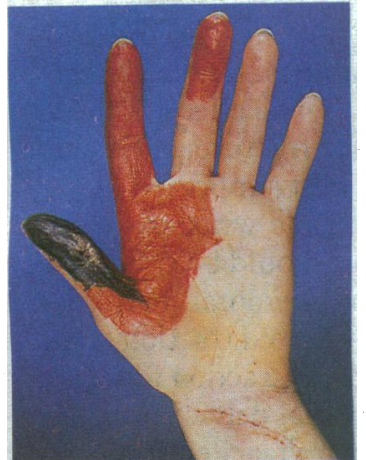

Areas of complete (black) and partial (red) sensory loss resulting from a wrist wound indicate damage to the median nerve, which was missed when the wound was sutured.

\section{Neurological state}

Evidence of nerve injury may be difficult to obtain in the unconscious or multiply injured patient. There is a higher incidence of neurological damage with dislocations than with fractures. Simple tests of sensitivity to touch, motor function, and sweating are sufficient to determine nerve integrity. When testing distal motor function the more proximal innervation of the muscle bellies must be appreciated. Division of a peripheral nerve must be assumed to have occurred if there is altered sensation in the distribution of that nerve and a wound overlying its course. Neurological function should be documented to allow later comparison.

\section{Wound management}

\author{
Management of wounds \\ (1) Obtain samples for culture \\ (2) Give preventive antibiotics intravenously \\ (3) Administer tetanus immunisation if \\ necessary \\ (4) Remove particulate contaminants by \\ physical wound cleaning and irrigation \\ (5) Excise all devitalised tissue \\ (6) Take a split skin graft early from skin flaps \\ of doubtful viability \\ (7) Anticipate swelling, decompress \\ compartments, and leave wounds open \\ (8) Obtain early rigid fracture stabilisation
}

\section{Pelvic fractures}

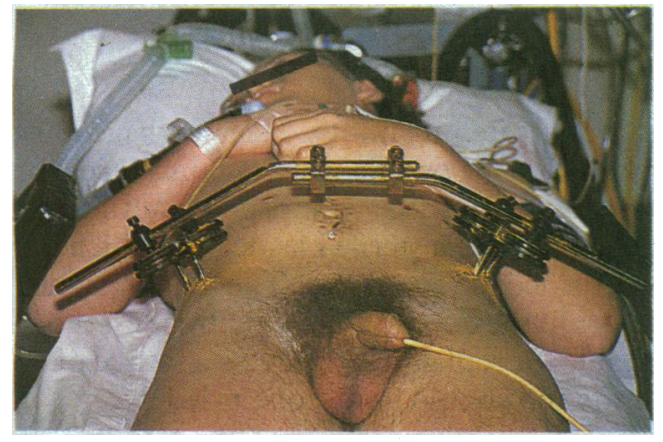

Pelvic external fixator.

\section{Radiology}

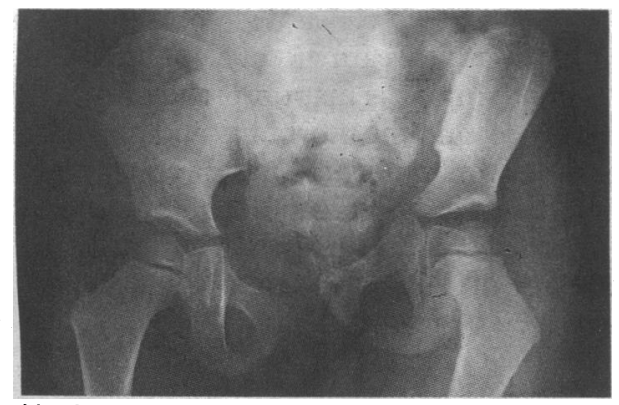

Vertical shear disruption of left hemipelvis.
Among patients with open fractures, $50 \%$ have multiple injuries. Correct management of wounds in the first few hours is decisive. The extent of the soft tissue damage will determine the outcome. A sterile dressing applied to an open wound at the site of the accident should not be disturbed. Definitive surgical toilet is required within six hours, and repeated examinations outside the operating theatre will considerably increase the risk of infection. Instant photography of the wound has been recommended to prevent this interference. As an adjunct to surgical toilet preventive antibiotics are indicated in patients with open fractures and contaminated wounds. A cephalosporin (for example, cefuroxime $750 \mathrm{mg}$ three times a day) and gentamicin ( $80 \mathrm{mg}$ three times a day) are appropriate and should be given intravenously for at least three days. Tetanus prophylaxis must not be forgotten and depends on the patient's previous immunisation state. An immunised patient with a contaminated wound that is prone to tetanus requires a booster dose of tetanus toxoid if more than five years have elapsed since his or her last dose. In addition, tetanus immunoglobulin is required if no immunity exists or the immunisation state is unknown.

Fractures of the pelvis are common in patients with multiple injuries and a radiograph is essential. The severity of these fractures is often underestimated: the fracture displacement visible in the radiograph may poorly reflect the disruption at the time of impact. A considerable force is necessary to disrupt the pelvic ring. The associated extensive soft tissue and visceral damage may result in life threatening haemorrhage. Damage to pelvic organs is common, particularly that involving the urinary tract. Urethral catheterisation should not be attempted if this is suspected because of the presence of blood at the urethral meatus, a perineal haematoma, or a high riding prostate on rectal examination. An urgent specialist opinion should be sought. Abdominal examination may be equivocal if there is a major pelvic fracture, and diagnostic peritoneal lavage may be useful. Urgent reduction and stabilisation with an external fixator will appreciably reduce blood loss in the patients with major pelvic disruptions.

Only when the multiply injured patient is resuscitated and stable and the three essential radiographs - of the chest, lateral cervical spine, and pelvis - have been performed should radiographs of limbs be considered. The standard two projections at right angles to one another are appropriate and must include the whole bone suspected of being fractured and the adjacent joints. Radiographs must be scrutinised for joint dislocations and subluxations that may be associated with fractures. Beware of requesting extensive radiographic studies if this requires the patient to be moved from the resuscitation area and its monitoring facilities. 


\section{Splintage}

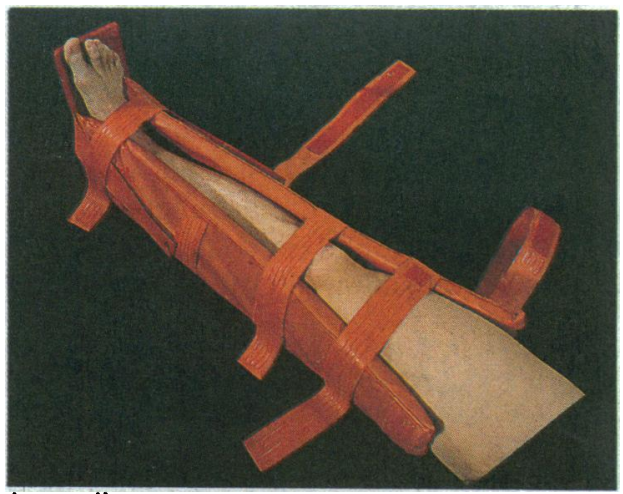

Leg splint.

\section{Compartment syndromes}

\section{Symptoms and signs of compartment syndrome}

- Increasing pain-despite immobilisation of fractures

- Altered sensation in the dermatome of the nerve(s) passing through that compartment

- Palpable raised tension and tenderness of the muscle compartment

- Pain on passively stretching the muscles within the compartment

Beware:

- The pulses are often present

- Maintain a high index of suspicion in the unconscious or anaesthetised patient

\section{Traumatic amputations}

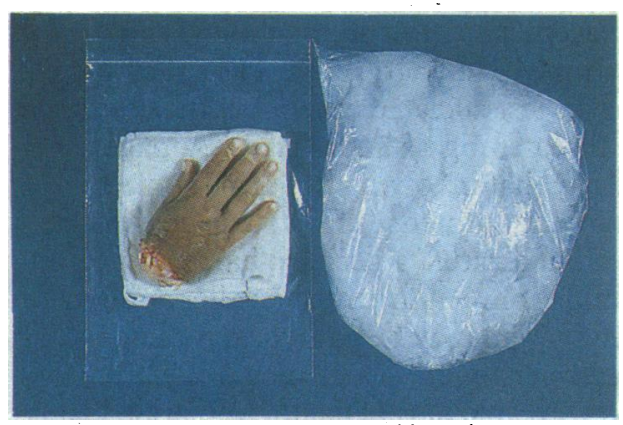

Management of an amputated hand.

\section{Definitive management of fractures}

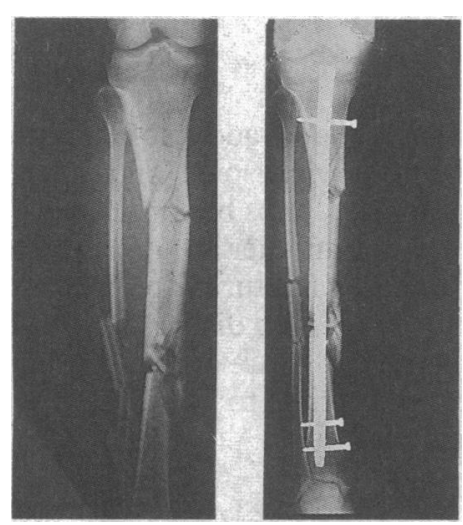

Internally fixed

fractured tibia.
The correct use of splintage will afford considerable pain relief, avert further soft tissue damage, and facilitate transport. To be effective the splint must immobilise the joint above and below the fracture and include the bone on either side of a dislocation. The arm is best supported by a simple sling and bandaged to the body. The forearm and wrist are immobilised on padded splints or pillows. The hand should be splinted in a functional position - that is, gripping a bandage roll. Femoral shaft fractures may be adequately controlled only by using fixed traction splints such as the Thomas splint or the modern equivalent. A traction force is applied to the leg or foot and is countered by a proximal pelvic bar. Low pressure $(30 \mathrm{~mm} \mathrm{Hg})$ inflatable double walled polyvinyl jacket splints are now commonly used to immobilise tibial, ankle, and forearm fractures they are easy to use and effective.

Multiply injured patients with reduced tissue perfusion and oxygenation are at high risk of developing compartment syndromes. Increasing swelling in the unyielding fascial compartments, particularly in the forearm and lower leg, as a result of tissue contusion, bleeding, or ischaemia may result in autoinfarction of the compartment muscle. The clinical symptoms and signs are increasing pain, sensory deficit in the distribution of the peripheral nerves passing through that compartment, progressive swelling and tension, and pain on passive muscle stretching. The presence of peripheral pulses does not exclude an evolving compartment syndrome.

If signs of a compartment syndrome develop all potentially constricting dressings, casts, and splints should be released. If rapid recovery is not observed then prompt fasciotomy should be performed. A high index of suspicion must be maintained in the unconscious patient and continuous instrumented compartment pressure monitoring may be indicated. Compartment pressures of greater than $30 \mathrm{~mm} \mathrm{Hg}$ are considered abnormal. It is a fallacy that compartment syndromes do not develop in patients with open fractures - an incidence of $15 \%$ has been reported.

Amputation is a catastrophic life threatening injury. Haemorrhage must be controlled as a priority. Replantation is possible in certain instances. In these cases the amputated part should be cleaned; wrapped in a sterile cloth that has been soaked in saline; and sealed in a sterile plastic bag, which is then immersed in a container of crushed ice and water. The limb must not be allowed to freeze. Rapid transfer to the definitive care centre is essential. Amputated parts that are unsuitable for replantation may be a source of bone, skin, vessel, and nerve grafts and should not be discarded.

\section{Closed fractures}

The option of either surgical or conservative treatment of closed fractures available in patients with an isolated limb injury is inappropriate in the multiply injured patient. The incidence of, and the morbidity and mortality associated with, the adult respiratory distress syndrome, fat embolism, and late systemic sepsis are considerably reduced if the major long bone fractures are rigidly stabilised by internal or external fixation within 24 hours. This also results in easier nursing of the patient and a reduction in requirements for narcotic analgesia. 

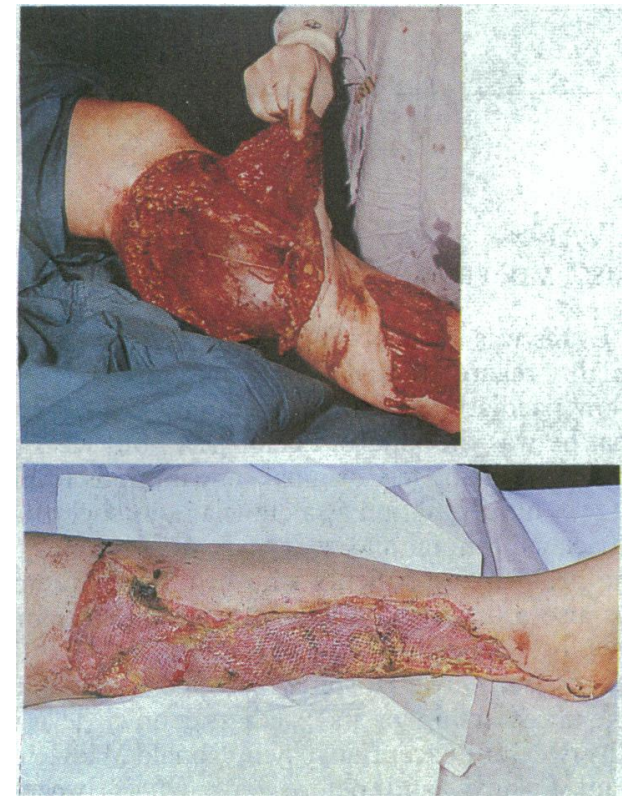

Soft tissue damage to the leg after a degloving injury (top); late flap necrosis was treated by excision and application of the split skin graft (meshed) obtained at the first operation (bottom).

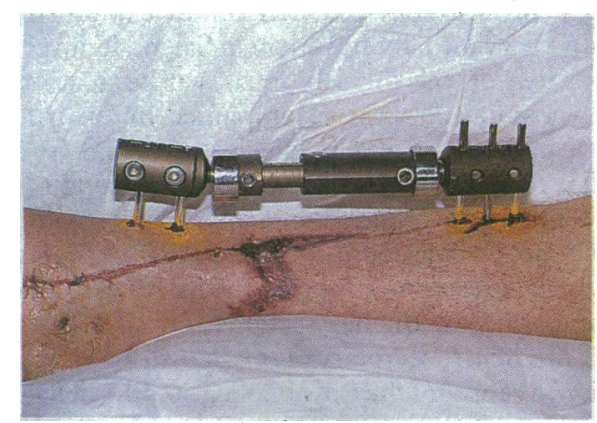

Externally fixed|fracture:

\section{Conclusions}

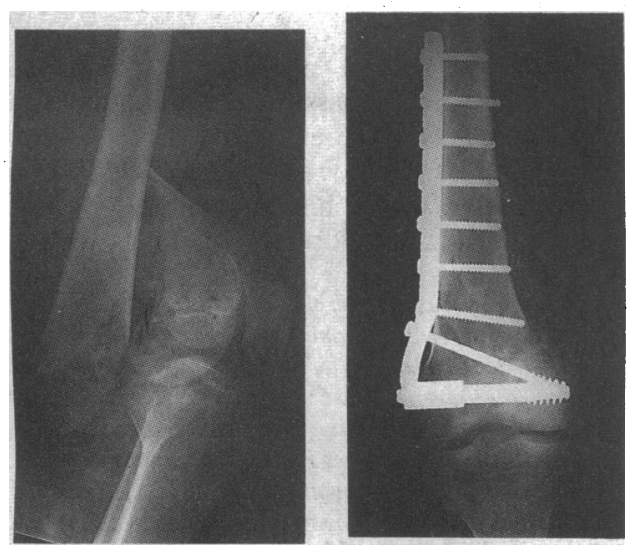

Unstable displaced supracondylar fracture of the femur (left); after internal fixation (right).

\section{Open fractures}

The soft tisue damage and the risk of infection are the two critical factors determining outcome in patients with serious open fractures of the limbs. The treatment employed in the first few hours can determine the difference between complete recovery and lifelong disability. In remembering that the infecting organisms are the contaminating organisms, samples for culture should be obtained at the outset. The priorities are to reduce the size of the infecting inoculum by physical cleaning and to ensure that all devitalised tissue is excised.

In a multiply injured patient with a reduced oxygen delivery and an anticipated rise in tissue pressure, wound hypoxia and an increased susceptibility to infection are inevitable. Closure of the wound is therefore rarely indicated.

Toilet of the wound is often performed inadequately. The surrounding skin should be shaved and particulate debris removed by scrubbing the wound with a brush. Meticulous exploration of the wound is necessary, and all recesses should be liberally irrigated by using a squirt and suck technique. Large volumes of warm saline or antiseptic solution are necessary (4-10 litres). Pressurised pulsed irrigation systems are now commercially available.

The wound is extended to facilitate examination as required. All nonviable muscle, fascia, and fat is carefully but radically excised. Fasciotomies may be performed once the wound has been thoroughly cleaned. Skin flaps of dubious viability are best dealt with by taking a split skin graft from the flap surface. This will serve to delineate the margin of viability, (the dead area will show no capillary bleeding). The harvested graft may be reapplied later if necrosis of the flap occurs. Small loose fragments of bone should be removed. Occasionally, large mechanically important fragments may be retained to help fracture fixation.

Most open fractures are unstable. Rigid stabilisation is now recognised to promote tissue healing. A variety of methods are employed; the application of an external skeletal fixator is currently the most popular; this facilitates wound access and nursing care. Closure of the skin defect within seven days with a split skin graft, myocutaneous flap, or microvascular free flap as appropriate will appreciably reduce the risk of infection and rate of non-union of these fractures.

The assessment and management of limb trauma should always be secondary to resuscitation and management of life threatening conditions. A knowledge of the mechanism of injury and careful examination are essential if all of the sustained injuries are to be identified. Assessment of the peripheral circulation is crucial to allow early detection and management of potentially limb threatening injuries. Appropriate reduction of fractures and dislocations combined with correct splintage will reduce pain and can prevent serious complications.

A high level of suspicion is necessary in the multiply injured patient to identify nerve injuries and detect evolving compartment syndromes.

Frequent reassessment and recordings of the circulation and neurological function of an injured limb are essential. The seriousness of open fracture wounds should be appreciated and an aggressive approach to wound toilet adopted. Urgent operative fixation of the major fractures within the first 24 hours will contribute to a reduction in mortality and morbidity.

The illustrations of leg trauma were reproduced by kind permission of $\mathrm{Mr} \mathrm{A} \mathrm{Cobb}, \mathrm{Mr}$ I Hudson, and $\mathrm{Mr} \mathrm{R}$ Birch, and the radiograph of the pelvic fracture by Dr D Stoker. The pictures of the leg splint and amputated hand were taken by the department of medical photography, Royal National Orthopaedic Hospital, London. The line drawings were prepared by the education and medical illustration services department, St Bartholomew's Hospital.

Mr Keith Willett, FRCS, is senior registrar in traumatic and orthopaedic surgery at Charing Cross Hospital; Mr Hugh Dorrell, FRCs, is consultant in orthopaedic surgery, and Mr Peter Kelly, FRCS, is consultant in accident and emergency medicine at Lister Hospital, Stevenage.

The ABC of Major Trauma has been edited by Mr David Skinner, FRCs; Mr Peter Driscoll, FRCS; and Mr Richard Earlam, FRCS. 